\title{
Response to inhibition of smoothened in diverse epithelial cancer cells that lack smoothened or patched 1 mutations
}

\author{
FABRIZIO GALIMBERTI ${ }^{1}$, ALEXANDER M. BUSCH ${ }^{1}$, FADZAI CHINYENGETERE ${ }^{1}$, TIAN MA ${ }^{1}$, DAVID SEKULA ${ }^{1}$, \\ VINCENT A. MEMOLI ${ }^{2,5}$, KONSTANTIN H. DRAGNEV ${ }^{4,5}$, FANG LIU ${ }^{1}$, KEVIN C. JOHNSON ${ }^{1}$, YONGLI GUO ${ }^{1}$, \\ SARAH J. FREEMANTLE ${ }^{1}$, ANGELINE S. ANDREW ${ }^{3,5}$, PATRICIA GRENINGER ${ }^{6}$, DAVID J. ROBBINS ${ }^{1,5}$, \\ JEFF SETTLEMAN $^{6}$, CYRIL BENES $^{6}$ and ETHAN DMITROVSKY ${ }^{1,4,5}$ \\ Departments of ${ }^{1}$ Pharmacology and Toxicology, ${ }^{2}$ Pathology, ${ }^{3}$ Community and Family Medicine, ${ }^{4}$ Medicine, and \\ ${ }^{5}$ The Norris Cotton Cancer Center, Geisel School of Medicine at Dartmouth, Hanover, NH 03755 and \\ Dartmouth-Hitchcock Medical Center, Lebanon, NH 03756; ${ }^{6}$ Center for Molecular Therapeutics, \\ Massachusetts General Hospital Cancer Center, Charlestown, MA 02129, USA
}

Received May 30, 2012; Accepted July 19, 2012

DOI: 10.3892/ijo.2012.1599

\begin{abstract}
Hedgehog (HH) pathway Smoothened (Smo) inhibitors are active against Gorlin syndrome-associated basal cell carcinoma (BCC) and medulloblastoma where Patched (Ptch) mutations occur. We interrogated 705 epithelial cancer cell lines for growth response to the Smo inhibitor cyclopamine and for expressed HH pathway-regulated species in a linked genetic database. Ptch and Smo mutations that respectively conferred Smo inhibitor response or resistance were undetected. Previous studies revealed $\mathrm{HH}$ pathway activation in lung cancers. Therefore, findings were validated using lung cancer cell lines, transgenic and transplantable murine lung cancer models, and human normal-malignant lung tissue arrays in addition to testing other Smo inhibitors. Cyclopamine sensitivity most significantly correlated with high cyclin $\mathrm{E}(\mathrm{P}=0.000009)$ and low insulin-like growth factor binding protein 6 (IGFBP6) $(\mathrm{P}=0.000004)$ levels. Gli family members were associated with response. Cyclopamine resistance occurred with high GILZ $(\mathrm{P}=0.002)$ expression. Newer Smo inhibitors exhibited a pattern of sensitivity similar to cyclopamine. Gain of cyclin E or loss of IGFBP6 in lung cancer cells significantly increased Smo inhibitor response. Cyclin E-driven transgenic lung cancers expressed a gene profile implicating $\mathrm{HH}$ pathway activation. Cyclopamine treatment significantly reduced proliferation of murine and human lung cancers. Smo inhibition reduced lung cancer formation in a syngeneic mouse model. In human normal-malignant lung tissue arrays cyclin E, IGFBP6, Gli1 and GILZ were each differentially expressed. Together, these
\end{abstract}

Correspondence to: Dr Ethan Dmitrovsky, Department of Pharmacology and Toxicology, Geisel School of Medicine at Dartmouth, Hanover, NH 03755, USA

E-mail: ethan.dmitrovsky@dartmouth.edu

Key words: hedgehog, smoothened, patched, lung cancer findings indicate that Smo inhibitors should be considered in cancers beyond those with activating $\mathrm{HH}$ pathway mutations. This includes tumors that express genes indicating basal $\mathrm{HH}$ pathway activation.

\section{Introduction}

Hedgehog $(\mathrm{HH})$ signaling is important in development and carcinogenesis because it controls cell fate and proliferation $(1,2)$. Three mammalian HH ligands [sonic (sHH), desert, and indian] are lipid-modified secreted proteins $(1,2)$. The $\mathrm{HH}$ receptor Patched 1 (Ptch1) inhibits this pathway by locking Smoothened (Smo) in an inactive conformation. After $\mathrm{HH}$ ligand treatment, Ptch1 releases Smo inhibition, which augments Gli family member (Gli1, Gli2, and Gli3) expression (1,2). Gli1 and Gli2 typically exert stimulatory while Gli3 has inhibitory effects (1). Activating the $\mathrm{HH}$ pathway affects expression of Ptch1, cyclin D1, cyclin E, insulin-like growth factor 2 (IGF2), insulin-like growth factor binding protein 6 (IGFBP6), GILZ and other species (2-5).

$\mathrm{HH}$ pathway deregulation occurs in many tumors including lung (6), breast (7), and pancreatic (8) cancers. Ptch1 mutations occur in the Gorlin syndrome-associated cancers basal cell carcinoma (BCC) and medulloblastoma (9-12). Smo inhibition can chemoprevent invasive BCC (13). Cyclopamine, a naturally-occurring $\mathrm{HH}$ antagonist, binds to Smo and inhibits HH signaling (14). Other Smo inhibitors exist with antineoplastic effects in vitro and in clinical trials for patients with BCC or medulloblastoma (9-12). The $\mathrm{HH}$ pathway regulates growth of small cell lung cancer (SCLC) and non-small cell lung cancer (NSCLC) $(6,15)$.HH pathway members are abundantly expressed in the premalignant and malignant lungs of cyclin E-expressing transgenic mice (16). Resistance to Smo inhibitors occurs with acquired Smo mutations $(17,18)$.

This study uncovered growth inhibitory responses to Smo inhibition in diverse cancer cells using a robotic-based platform with a genetic database. In this database Ptch1 and Smo sequences were available with information about expression of 
species associated with $\mathrm{HH}$ pathway activation. Basal expression of these species in cancer cells was hypothesized to indicate growth dependence of these cells on the $\mathrm{HH}$ pathway. It was hypothesized that cancer cells expressing these species would respond to a Smo inhibitor.

Multiple Smo inhibitors were studied in lung cancer because the $\mathrm{HH}$ pathway is active in subsets of these cancers. Both murine and human lung cancer cell lines exist. Cyclin E-driven transgenic and transplantable murine lung cancer models that spontaneously activated the $\mathrm{HH}$ pathway were available for study as was a paired human normal-malignant lung tissue array with an associated clinical database. The presented findings implicate use of Smo inhibitors for lung and other cancers when a gene profile indicative of $\mathrm{HH}$ pathway dependence is expressed in the cancer cells.

\section{Materials and methods}

Cell culture. ED-1 and ED-2 murine lung cancer lines, C-10 murine immortalized lung epithelial cells, BEAS-2B human immortalized bronchial epithelial cells, and human lung cancer cell lines (A549, HOP-62, H-522, U-1752, NCI-H1730, and NCI-H2122) were each cultured in RPMI-1640 medium with $10 \%$ fetal bovine serum (FBS) and $1 \%$ antibiotic and antimycotic solution at $37^{\circ} \mathrm{C}$ in $5 \% \mathrm{CO}_{2}$ in a humidified incubator, as before $(15,16,19-21)$. Cell lines were obtained from and authenticated (using genotypic and phenotypic assays) by ATCC except for murine ED-1 and ED-2 lung cancer cell lines that were previously described and authenticated $(19,21)$.

Chemicals. Cyclopamine (LC Laboratories, Wobrun, MA) and tomatidine (Sigma-Aldrich, St. Louis, MO) were purchased as were recombinant mouse sHH (R\&D Systems, Minneapolis, $\mathrm{MN}$ ) and FBS (Gemini Bioproducts, Inc, Calabasas, CA). The Smo inhibitor MK-4101 (22) was provided by Merck. The SANT-1 Smo inhibitor (15) was purchased (Tocris Bioscience, Ellisville, MO) as was the SAG Smo agonist (EMD Millipore, Billerica, MA).

Repression of HH pathway members. Cells were independently treated with the Smo inhibitors: cyclopamine, SANT-1 and MK-4101. In vivo Smo inhibition was achieved in mouse lung cancer models with cyclopamine (intraperitoneal injections, $40 \mathrm{mg} / \mathrm{kg}$ ) treatments or with short hairpin RNA (shRNA)-mediated Smo knock-down in ED-1 cells. Individual small interfering RNA (siRNA)-mediated or shRNA-mediated repression of Gli1, Gli2, or Gli3 was achieved.

High-throughput proliferation assays. Cyclopamine growth effects were investigated in 705 human cancer cell lines using a high-throughput screen $(19,23,24)$. Cells were treated with cyclopamine at $10 \mu \mathrm{M}$ (and lower dosages) in media with 5\% FBS and were assayed at $72 \mathrm{~h}$ with quantification by the SpectraMax M5 plate reader (Molecular Devices, Sunnyvale, CA). Means of triplicate cyclopamine treatment experiments were compared to vehicle controls, using optimized methods $(19,23,24)$.

Smo inhibitor responses. The HH pathway affects expression of Ptch1, cyclin D1, cyclin E, IGF2, IGFBP6, GILZ, Gli family members, and other species (2-5). The cor.test function (25) of
$\mathrm{R}$ (26) compared cyclopamine-dependent growth responses to expressed species. Expression values were from U133 Plus 2.0 Affymetrix arrays and are publically available (27). The data set consisted of 490 samples corresponding to 164 unique cell lines that were typically examined in triplicate. Correlations were done: a) using all samples and b) using samples with a cyclopamine growth inhibitory response $\leq 0.75$ at the $10 \mu \mathrm{M}$ dosage, corresponding to 110 samples (in triplicate). Cyclopaminemediated growth response was compared with mutation data available from the Sanger database (28).

Ptchl and smo sequence analyses. Sequencing of the coding regions of Ptch1 was performed as before (29). For murine cell lines, murine-specific primers were used to sequence homologous domains of Ptch1. To assess for Smo mutations in murine and human cell lines, prior mutations in human Smo were searched for (A324T, V404M, D473H, E518K, W535L, and T640A) and corresponding regions of murine Smo were sequenced.

Transient transfection, proliferation and apoptosis assays. Logarithmically growing ED-1 $\left(3 \times 10^{4}\right.$ and $\left.4.5 \times 10^{3}\right)$, ED-2 $\left(8 \times 10^{4}\right)$, C-10 $\left(1 \times 10^{5}\right)$, BEAS-2B $\left(3 \times 10^{5}\right)$, A549 $\left(5 \times 10^{4}\right)$, HOP-62 $\left(5 \times 10^{4}\right), \mathrm{H}-522\left(3 \times 10^{5}\right), \mathrm{U} 1752\left(5 \times 10^{4}\right), \mathrm{NCI}-\mathrm{H} 1703\left(4.5 \times 10^{5}\right)$, and NCI-H2122 $\left(6 \times 10^{5}\right)$ cells were individually plated onto each well of 6-well tissue culture plates (BD Bioscience, San Jose, CA) $24 \mathrm{~h}$ before transfection or drug treatments. Three replicate experiments were performed in triplicate. Logarithmically growing cells were assayed using the CellTiter-Glo assay (Promega, Madison, WI) (21). Apoptosis was scored with the Caspase-Glo 3/7 Assay System (Promega).

SiRNA transfections were with Oligofectamine reagent (Invitrogen, Carlsbad, CA). SiRNAs targeting Gli1, Gli2, Gli3, Smo, IGFBP6 or a RISC-free control were each purchased (Dharmacon, Lafayette, CO). Two different siRNAs independently targeted Gli1, Gli2, Gli3, Smo, or IGFBP6 sequences: 5'-GGUUGGAACUUCUGUGAUG-3' (Gli1.1), 5'-GAGCAGG CCUCCGUUGUA-3' (Gli1.2), 5'-GGGAGAAGAAGGAGUU CGU-3' (Gli2.1), 5'-GGUUUGUGGUUGAGCGGAA-3' (Gli2.2), 5'-CCAUCGGUGGAAAAGCGU-3' (Gli3.1), 5'-GAA ACGCAAUCACUAUGCA-3' (Gli3.2), 5'-AGAACCCGCUGU UCACCGA-3'(Smo1.1), 5'-GCAUCUGUUUUGUAGGCUA-3' (Smo1.2), 5'-CAUCGAGGCUUCUACCGGA-3' (IGFBP6-1), and 5'-CAACAGAGGAAUCCAGGCA-3' (IGFBP6-2). Transient transfections were with FuGENE 6 reagent (Roche Applied Science, Indianapolis, IN). Reporter assays were performed after transfection of a GLIBS-Luciferase reporter construct or TK-Luciferase control plasmid (19). The DualLuciferase reporter assay system (Promega) was used in three replicate experiments, each performed in triplicate.

Stable knock-down. ED-1 cells stably expressing green fluorescent protein (GFP) were individually infected with lentivirus expressing shRNAs targeting Smo: TRCN0000026245 (Smol) and TRCN0000026295 (Smo2); IGFBP6: TRCN0000114766 (IGFBP6.1) and TRCN0000114768 (IGFBP6.2); GILZ: TRCN0000085743 (GILZ.1) and TRCN0000085746 (GILZ.2), or a scrambled control shRNA (RHS4080) (Open Biosystems, Huntsville, AL). Selection was with puromycin $(5 \mu \mathrm{g} / \mathrm{ml})$. Knock-downs were determined using semi-quantitative realtime reverse transcription (RT) polymerase chain reaction (PCR) 
assays. Cells with the most robust Smo knock-down versus controls were selected for tail vein injection experiments into syngeneic mice $(19,20,30)$.

Real-time RT-PCR assays. Total RNA was isolated using the RNeasy kit (Qiagen, Valencia, CA). RT was with the High Capacity cDNA RT kit (Applied Biosystems, Foster City, CA) and a Peltier Thermal Cycler (MJ Research, Waltham, MA). Semi-quantitative real-time RT-PCR assays were with SYBR Green PCR Mastermix (Applied Biosystems) and the 7500 Fast real-time PCR system (Applied Biosystems). Human IGFBP6 and $\beta$-actin qPCR assays were performed with TaqMan Universal PCR Master Mix and the manufacturer's described assays (Applied Biosystems). Three replicate experiments were performed. Primers were: murine Glil forward: 5'-CCAAGC CAACTTTATGTCAGGG-3', and reverse: 5'-AGCCCGCTTCT TTGTTAATTTGA-3'; murine Gli2 forward: 5'-CAACGCCTA CTCTCCCAGAC-3', and reverse: 5'-GAGCCTTGATGTACT GTACCAC-3'; murine Gli3 forward: 5'-CACAGCTCTACGGC GACTG-3', and reverse: 5'-CTGCATAGTGATTGCGTTTC TTC-3'; murine Smo forward: 5'-GAGCGTAGCTTCCGGGA CTA-3', and reverse: 5'-CTGGGCCGATTCTTGATCTCA-3'; murine IGFBP6 forward: 5'-TGCTAATGCTGTTGTTCG CTG-3', and reverse: 5'-CACGGTTGTCCCTCTCTCCT-3'; murine GILZ forward: 5'-ACCACCTGATGTACGCTGTG-3', and reverse: 5'-TCTGCTCCTTTAGGACCTCCA-3'; murine glyceraldehyde 3-phosphate dehydrogenase (GAPDH) forward: 5'-AGGTCGGTGTGAACGGATTTG-3', and reverse: 5'-TGT AGACCATGTAGTTGAGGTCA-3'; human Gli1 forward: 5'-GGCACCATGAGCCCATCTC-3', and reverse: 5'-ATCACC TTCCAAGGGTTCCTC-3'; human Gli2 forward: 5'-GGTGA AGCCTCCACCCTTTC-3', and reverse: 5'-TGCATGTAGTTT ACCCTGGGG-3'; human Gli3 forward: 5'-CTCCACGACC ACTGAAAAGAAA-3', and reverse: 5'-TCTCTGTGATAAG TCTGTCCAGG-3'; human Smo forward: 5'-GGCAACAGC ATTGCAGTGAAG-3', and reverse: 5'-GAGGAGAGACACA CGAGCCT-3'; and human GAPDH forward: 5'-ATGGGGAA GGTGAAGGTCG-3', and reverse: 5'-GGGGTCATTGATG GCAACAATA-3'.

Clonal growth assays. Two hundred logarithmically growing ED-1 cells were plated for triplicate replicate clonal growth assays, as before (20). Colonies were stained with Diff Quick (IMEB Inc, San Marcos, CA) and were counted with the Oxford Optronix Col Count counter (Oxford Optronix, Oxford, UK) (31).

Normal-malignant lung tissue array. The New Hampshire State Cancer Registry and the Dartmouth-Hitchcock Medical Center Tumor Registry have been described (20). Analyses of normal versus malignant lung tissue arrays were by a pathologist (Vincent A. Memoli) unaware of findings from the associated clinical database. Signed consents were obtained; studies were reviewed and approved by Dartmouth's Institutional Review Board (IRB) for human subjects. A paired normal-malignant lung tissue microarray was constructed (20).

Statistical analysis. Results were expressed as means \pm standard deviations. Results of all independent experiments were pooled to assess for statistical significance. Z-test and two-sided t-tests were used for statistical analyses with significance considered for values of $\mathrm{P}<0.05$.

Cyclopamine treatments of transgenic mice. Three 9-month-old female mice expressing transgenic wild-type human cyclin $\mathrm{E}$ were each treated daily (intraperitoneal) for 5 consecutive days with cyclopamine (40 mg/kg) or vehicle (45\% hydroxypropylB-cyclodextrin, HBS) with a total of six mice studied. Mice were examined using an Institutional Animal Care and Use Committee (IACUC)-approved protocol. Tissues were formalinfixed, paraffin-embedded and sectioned for histopathology (20). Hematoxylin and eosin staining as well as Ki-67, cyclin D1, and cyclin $\mathrm{E}$ immunostaining were performed as before $(16,19)$. Analyses were by a pathologist (Vincent A. Memoli) unaware whether harvested tissues were from cyclopamine or vehicletreated mice.

Murine syngeneic lung cancer transplantation assays. ED-1 cells were transduced with a GFP expression vector and transductants were sorted $(15,16)$ with $8 \times 10^{5}$ cells injected into tail veins of each female FVB mouse (8-week old). Ten mice per arm were used and replicate experiments were performed. To investigate cyclopamine antineoplastic effects, 10 mice were each intraperitoneally treated daily for 14 days with cyclopamine $(40 \mathrm{mg} / \mathrm{kg}) ; 10$ additional mice were each treated with vehicle (45\% HBS). Treatments began 2 weeks after tail-vein injections because lung tumors formed by then (data not shown). Ten syngeneic FVB mice were each injected with ED-1 cells that were treated with either MK-4101 $(10 \mu \mathrm{M})$ or vehicle as part of an IACUC-approved protocol. Mice were sacrificed 28 days post-tail vein injections. Replicate experiments were performed. Lung tissues were formalin-fixed, paraffin-embedded and sectioned for histopathology $(16,19,20)$. A rabbit polyclonal anti-GFP antibody (product ab290) (Abcam, Cambridge, MA) was used to identify lung tumors. Hematoxylin counterstaining was used.

\section{Results}

Growth effects of a Smo inhibitor were studied using a roboticbased platform linked to a genetic database $(19,23,24)$. A total of 705 different human cancer lines were examined for responses to the Smo inhibitor cyclopamine $(10 \mu \mathrm{M})$. Proliferation was reduced in a subset of cancer cell lines after $72 \mathrm{~h}$ of treatment (Fig. 1A, left panel). Of these lines, 353 were displayed in the Sanger database. No Ptch1 or Smo mutations were detected (3 lines had Smo amplifications that did not confer cyclopamine hypersensitivity). Data from the Sanger database showed the distribution of somatic mutations in Ptch1 for 860 unique cancer cell lines. Six had variant sequences of uncertain functional consequences. For the Smo gene, only 6 out of 866 different cancer cells had sequence variants of uncertain functional consequence. There were 152 lung cancer cell lines analyzed. None of these lines had Ptch1 or Smo mutations.

The $\mathrm{HH}$ pathway can affect expression of the Gli family members, Ptch1, cyclin D1, cyclin E, IGF2, IGFBP6, or GILZ (1-5). Cyclopamine-dependent growth responses were compared to these expressed species using the described database. Most highly significant associations occurred for high cyclin $\mathrm{E}$ $(\mathrm{P}=0.000009)$ and low IGFBP6 ( $\mathrm{P}=0.000004)$ levels (Fig. 1A). 
A

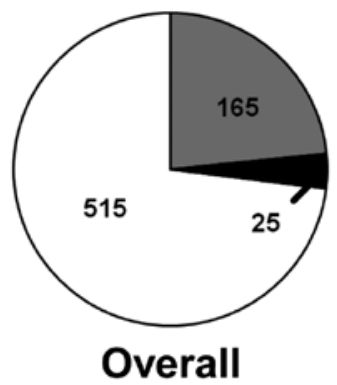

B

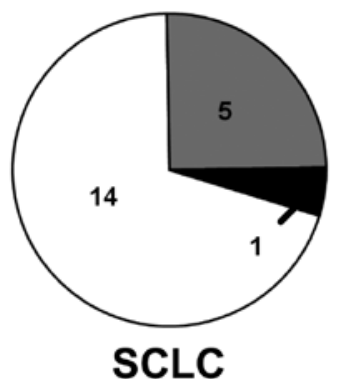

C

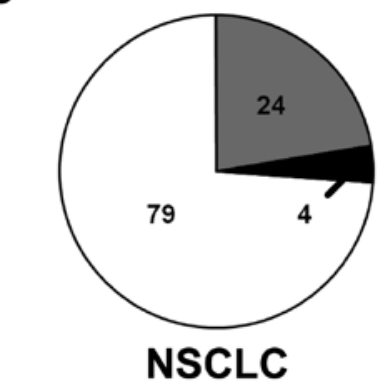

Fractional Growth

$<0.5 \square 0.5-0.8 \square>0.8$

\begin{tabular}{|c|c|c|}
\hline Gene & Correlation & P Value \\
\hline Cyclin E & High Levels $~$ Sensitive & $\mathbf{8 . 7 \times 1 0 ^ { - 5 }}$ \\
\hline IGFBP6 & Low Levels $~$ Sensitive & $4.0 \times 10^{-5}$ \\
\hline GILZ & High Levels $~$ Resistant & 0.002 \\
\hline Gli1 & High Levels $~$ Sensitive & $\mathbf{0 . 0 4}$ \\
\hline
\end{tabular}

Fractional Growth

$<0.5 \square 0.5-0.8 \square>0.8$

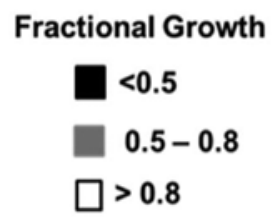

\begin{tabular}{|l|c|}
\hline \multicolumn{1}{|c|}{ Cell Line } & $\begin{array}{c}\text { Fractional } \\
\text { Growth }\end{array}$ \\
\hline DMS 53 & 0.797 \\
\hline SBC-5 & 0.617 \\
\hline DMS 273 & 0.606 \\
\hline RERF-LC-MA & 0.575 \\
\hline KHM-3S & 0.534 \\
\hline SBC-3 & $\mathbf{0 . 2 6 6}$ \\
\hline
\end{tabular}

\begin{tabular}{|l|c|l|c|}
\hline \multicolumn{1}{|c|}{ Cell Line } & $\begin{array}{c}\text { Fractional } \\
\text { Growth }\end{array}$ & Cell Line & $\begin{array}{c}\text { Fractional } \\
\text { Growth }\end{array}$ \\
\hline $\mathrm{NCl}-\mathrm{H} 1650$ & 0.799 & LU65B & 0.698 \\
\hline LUDLU-1 & 0.791 & $\mathrm{NCl}-\mathrm{H} 2170$ & 0.673 \\
\hline VMRC-LCD & 0.785 & $\mathrm{COR}-\mathrm{L} 23$ & 0.655 \\
\hline $\mathrm{A} 549$ & 0.785 & $\mathrm{NCl}-\mathrm{H} 1573$ & 0.642 \\
\hline $\mathrm{NCl}-\mathrm{H} 1581$ & 0.784 & $201 \mathrm{~T}$ & 0.629 \\
\hline $\mathrm{NCl}-\mathrm{H} 2030$ & 0.779 & $\mathrm{NCl}-\mathrm{H} 3122$ & 0.623 \\
\hline $\mathrm{NCl}-\mathrm{H} 2291$ & 0.775 & $\mathrm{NCl}-\mathrm{H} 2172$ & 0.582 \\
\hline $\mathrm{NCl}-\mathrm{H} 2228$ & 0.759 & $\mathrm{NCl}-\mathrm{H} 1944$ & 0.567 \\
\hline $\mathrm{NCl}-\mathrm{H} 2347$ & 0.75 & $\mathrm{Calu}-1$ & 0.557 \\
\hline $\mathrm{RERF}-\mathrm{LC}-\mathrm{Sq} 1$ & 0.735 & $\mathrm{NCl}-\mathrm{H} 1299$ & 0.548 \\
\hline $\mathrm{NCl}-\mathrm{H} 322$ & 0.733 & $\mathrm{PC}-9$ & $\mathbf{0 . 4 1 9}$ \\
\hline $\mathrm{NCl}-\mathrm{H} 650$ & 0.73 & $\mathbf{S W} \mathbf{9 0 0}$ & $\mathbf{0 . 3 2 6}$ \\
\hline $\mathrm{LU} 65 \mathrm{~A}$ & 0.728 & $\mathrm{NCl}-\mathrm{H} 1703$ & $\mathbf{0 . 3 1 9}$ \\
\hline $\mathrm{NCl}-\mathrm{H} 661$ & 0.724 & $\mathrm{NCl}-\mathrm{H2122}$ & $\mathbf{0 . 1 2 4}$ \\
\hline & & &
\end{tabular}

Figure 1. Cyclopamine-mediated growth inhibition of diverse human epithelial cancer cell lines. (A) Overall profile of cyclopamine (10 $\mu \mathrm{M})$-mediated growth response was interrogated in 705 cancer cell lines (left panel). The right panel displayed significant associations between growth inhibitory responses to cyclopamine and expressed HH pathway regulated species (right panel). (B) Cyclopamine-mediated growth responses in 20 small cell lung cancer (SCLC) cell lines. The 6 most growth inhibited lines are shown. (C) Cyclopamine-mediated growth inhibitory responses for 107 non-small cell lung cancer (NSCLC) lines. The 28 most growth inhibited lines are shown. Fractional growth responses for all panels (A, B and C) are displayed. Bold text indicated the most cyclopamine-responsive cells.

Less significant associations were detected for IGF2 $(\mathrm{P}=0.005)$ cyclin D1 ( $\mathrm{P}=0.024)$, Gli1 ( $\mathrm{P}=0.04)$, and Gli2 $(\mathrm{P}=0.05)$. High GILZ levels were linked to reduced cyclopamine response $(\mathrm{P}=0.002)$.

Twenty lines were of SCLC and 107 were of NSCLC origins. Subsets of SCLC (Fig. 1B) and NSCLC (Fig. 1C) lines responded to cyclopamine with substantial growth inhibition. NSCLC responses occurred independently of mutations for Ptch1 or Smo (Table IA). These responses were also independent of ras, epidermal growth factor receptor $(E G F R)$, or $p 53$ mutations (Table IB). $K$-RAS mutations in NSCLC are associated with resistance to EGFR-tyrosine kinase inhibitors (TKIs) (32-35). Growth inhibition in the most cyclopamine-responsive NSCLC cells lines (NCI-H1703 and NCI-H2122) were independently confirmed in Fig. 2.

Studies were next conducted in lung cancer because an autocrine $\mathrm{HH}$ pathway is reported in lung cancer (36). $\mathrm{HH}$ signaling is important for growth of NSCLC and SCLC $(6,15)$. Other reasons for studying lung cancer included the finding that increased cyclin E expression was associated with cyclopamine response (Fig. 1A) and cyclin E transgenic mice recapitulated key features of human lung cancer biology $(16,30)$.

Gain or loss of expression was achieved for the most significantly associated candidate regulators of cyclopamine response (cyclin E and IGFBP6) or resistance (GILZ). Gli1 is an established $\mathrm{HH}$ pathway regulator in lung cancer (15). To determine whether cyclin $\mathrm{E}$ affected cyclopamine response, cyclin $\mathrm{E}$ was overexpressed in murine immortalized C-10 lung epithelial cells (Fig. 2A, left panel shows a 2.26-fold increase in cyclin E protein versus actin expression) and this significantly $(\mathrm{P}<0.01)$ increased cyclopamine-mediated growth inhibition versus the inactive isomer tomatidine (Fig. 2A, right panel). Similar results were obtained using a second Smo inhibitor, SANT-1 (data not shown), which was consistent with prior studies (15). 
A
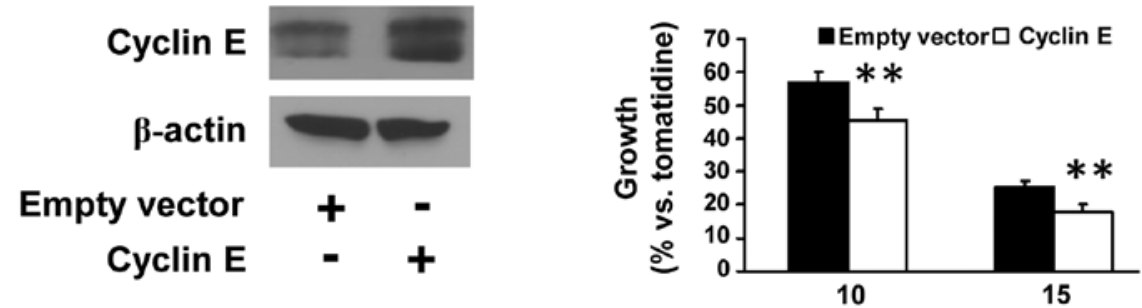

B
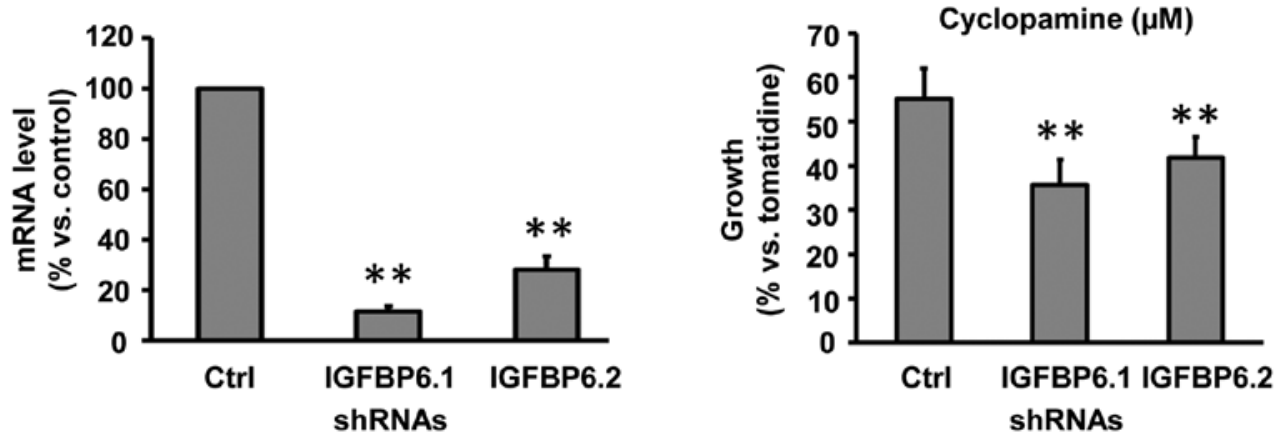

C
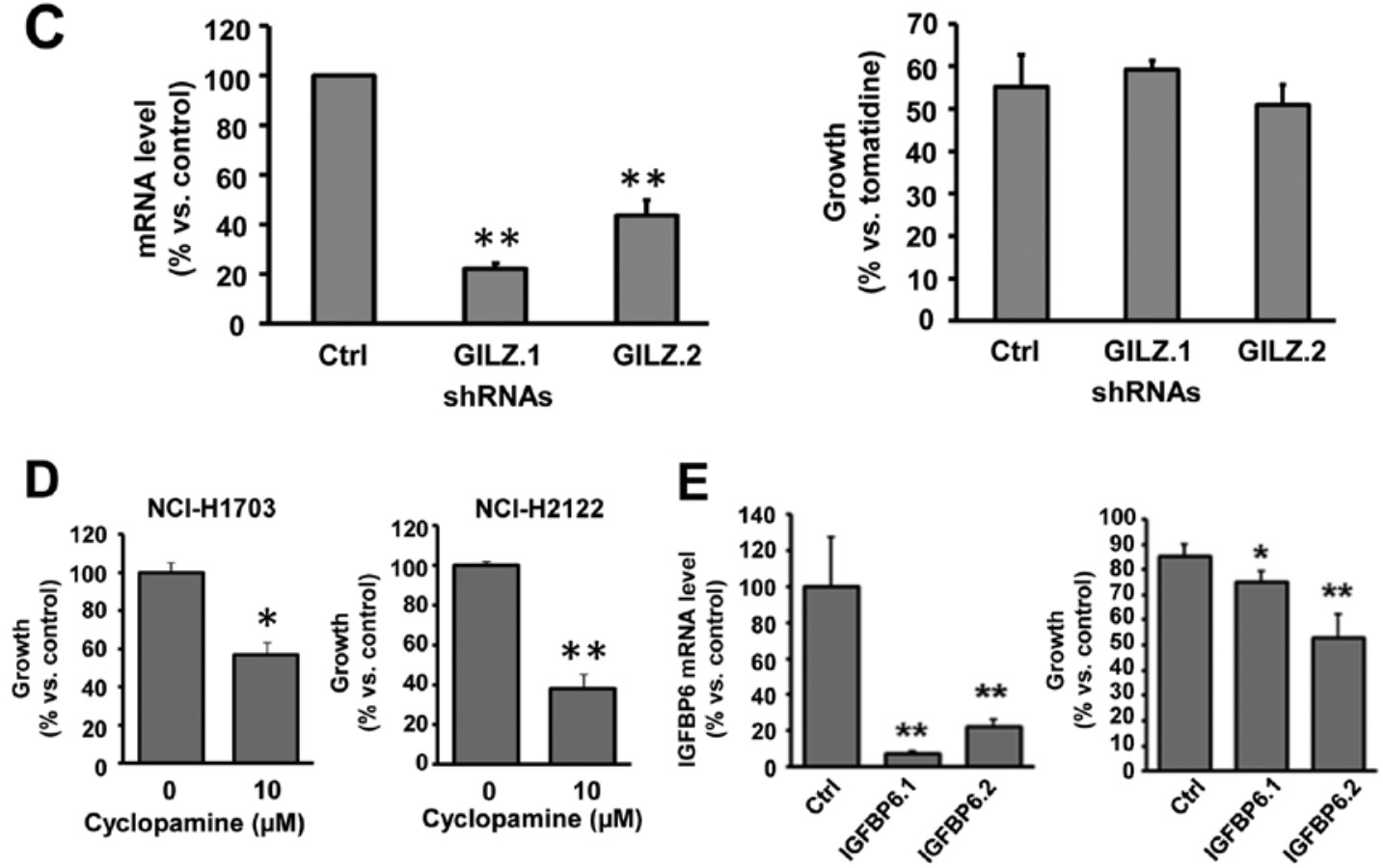

Figure 2. Functional validation of species associated with Smo-mediated growth inhibition. (A) Cyclin E was overexpressed in C-10 murine immortalized lung epithelial cells (left panel). Cyclin E overexpression enhanced response to cyclopamine treatment versus tomatidine controls (right panel). (B) IGFBP6 knock-down in ED-1 lung cancer cells (left panel) increased response to cyclopamine treatment versus controls (ctrl) (right panel). (C) GILZ knock-down in ED-1 cancer cells (left panel) did not significantly affect cyclopamine response (right panel). (D) Independent treatments of the human lung cancer cell lines NCI-H1703 (left panel) and NCI-H2122 (right panel) with cyclopamine $(10 \mu \mathrm{M})$ for three days reduced cell growth versus vehicle control. Basal IGFBP6 levels were at the lowest limit of detection by real-time PCR assays in NCI-H1703 cells (data not shown), consistent with the enhanced sensitivity of these cells to cyclopamine. (E) Higher IGFBP6 levels were detected in NCI-H2122 relative to NCI-H1703 cells. When IGFBP6 expression was knocked-down by transfection of two independent siRNAs (left panel), the sensitivity of NCI-H2122 to cyclopamine (10 $\mu \mathrm{M})$ was enhanced (right panel). Standard deviation bars are shown. ${ }^{*} \mathrm{P}<0.05 ;{ }^{* *} \mathrm{P}<0.01$.

Two shRNAs (Fig. 2B, left panel) were selected to reduce IGFBP6 expression independently in ED-1 lung cancer cells derived from a transgenic mouse that expressed wild-type cyclin E (30). Individual knock-down with these shRNAs significantly $(\mathrm{P}<0.01)$ increased Smo-mediated growth inhibition in this murine lung cancer cell line versus an inactive shRNA control (Fig. 2B, right panel). Thus, gain of cyclin E or loss of IGFBP6 expression regulated response to a Smo inhibitor. In contrast, two different shRNAs independently repressed GILZ expression (Fig. 2C, left panel), but these did not appreciably affect cyclopamine-mediated growth suppression at $72 \mathrm{~h}$ (Fig. 2C, right panel). Findings were confirmed in human NSCLC cells engineered with IGFBP6 knock-down (Fig. 2D and E).

Lung cancers arising in transgenic mice from increased cyclin E expression in the lung exhibit high levels of Gli1 $(16,30)$. These transgenic lung cancers are also shown to express low levels of IGFBP6 and GILZ versus the adjacent normal lung 
Table I. The (A) Ptch1 and Smo mutation status and (B) ras, p53, and activating EGFR mutation status of the 20 most cyclopamine-sensitive NSCLC cell lines examined in this study.

\begin{tabular}{lll} 
A, Ptch1 and Smo mutation status & \\
Cell line & Ptch1 status & Smo status \\
\hline NCI-H2122 & Wild-type & Wild-type \\
NCI-H1703 & Wild-type & Wild-type \\
SW900 & Wild-type & Wild-type \\
PC-9 & Not known & Not known \\
NCI-H1299 & Wild-type & Wild-type \\
Calu-1 & Wild-type & Wild-type \\
NCI-H1944 & Not known & Not known \\
NCI-H2172 & Not known & Not known \\
NCI-H3122 & Not known & Not known \\
201T & Not known & Not known \\
NCI-H1573 & Wild-type & Wild-type \\
COR-L23 & Wild-type & Wild-type \\
NCI-H2170 & Wild-type & Wild-type \\
LU65B & Not known & Not known \\
NCI-H611 & Wild-type & Wild-type \\
LU65A & Not known & Not known \\
NCI-H650 & Wild-type & Wild-type \\
NCI-H322 & Wild-type & Wild-type \\
RERF-LC-Sq1 & Not known & Not known \\
NCI-H2347 & Wild-type & Wild-type
\end{tabular}

$\mathrm{B}$, ras, p53, and activating EGFR mutation status

\begin{tabular}{llcl} 
Cell line & \multicolumn{1}{c}{ Ras status } & EGFR status & p53 status \\
\hline NCI-H2122 & Mutant (K-RAS) & Wild-type & Mutant \\
NCI-H1703 & Wild-type & Wild-type & Mutant \\
SW900 & Mutant (K-RAS) & Wild-type & Mutant \\
PC-9 & Wild-type & Mutant & Mutant \\
NCI-H1299 & Mutant (N-RAS) & Wild-type & Wild-type \\
Calu-1 & Mutant (K-RAS) & Wild-type & Wild-type \\
NCI-H1944 & Mutant (K-RAS) & Not known & Wild-type \\
NCI-H2172 & Not known & Not known & Not known \\
NCI-H3122 & Not known & Wild-type & Not known \\
201T & Wild-type & Wild-type & Not known \\
NCI-H1573 & Mutant (K-RAS) & Wild-type & Mutant \\
COR-L23 & Mutant (K-RAS) & Wild-type & Wild-type \\
NCI-H2170 & Wild-type & Wild-type & Mutant \\
LU65B & Not known & Not known & Mutant \\
NCI-H611 & Wild-type & Wild-type & Mutant \\
LU65A & Not known & Not known & Mutant \\
NCI-H650 & Mutant (K-RAS) & Wild-type & Mutant \\
NCI-H322 & Wild-type & Wild-type & Mutant \\
RERF-LC-Sq1 & Not known & Wild-type & Not known \\
NCI-H2347 & Mutant (N-RAS) & Wild-type & Wild-type \\
\hline
\end{tabular}

(Fig. 3A). Whether cell lines derived from these murine lung cancers responded to a Smo inhibitor was independently studied in ED-1 and ED-2 lung cancer cells derived respectively from transgenic mice whose lung tumors expressed wild-type (ED-1) or degradation-resistant (ED-2) cyclin E species (30).

Effects of Smo inhibition were explored by targeting Smo in ED-1 cells. Gli1, Gli2, Gli3, and Smo were independently targeted using two different siRNAs engineered to repress each of these species. Independent knock-down of Gli1, Gli2, and Smo each significantly inhibited ED-1 cell growth (as did cyclopamine treatment), but Gli3 knock-down did not appreciably affect growth (Fig. 3B, left and middle panels). As expected, the Smo agonist SAG augmented Glil expression; cyclopamine co-treatment prevented this (Fig. 3B, right panel). Compensatory changes in expressed $\mathrm{HH}$ pathway members were found after these different knock-downs in Fig. 3C and D. Smo knock-down substantially decreased lung cancer cell growth.

Cyclopamine treatment also significantly $(\mathrm{P}<0.01)$ decreased ED-1 clonal growth while increasing apoptosis (Fig. 4A). ED-1 and ED-2 cells expressed multiple $\mathrm{HH}$ pathway components as did the examined murine and human immortalized pulmonary epithelial or cancer cell lines (Fig. 4B). Genomic DNA from each of these cell lines was sequenced for the entire coding region of Ptch1 and for Smo mutations in regions that conferred Smo inhibitor resistance (A324T, V404M, D473H, E518K, W535L, and T640A). No mutations were detected (data not shown). Human lung cancer cell lines studied were those in which responses to Smo inhibition were already reported $(15,36)$. To establish whether the HH pathway was functional in ED-1 cells, these cells were treated with recombinant $\mathrm{sHH}$ at different dosages in low serum $(0.5 \%)$ containing medium. $\mathrm{HH}$ pathway activity in these cells was confirmed; $\mathrm{sHH}$ treatment significantly $(\mathrm{P}<0.01)$ increased mRNA expression for Gli1 and Ptch (Fig. 4C). Although ED-1 cells arose from transgenic lung cancers that activated the HH pathway (16), these cells had relatively low basal Gli1 and Gli2 mRNA levels (Fig. 4B) and low basal Gli1-reporter activity (Fig. 4D). This is consistent with differences reported between ex vivo versus in vivo $\mathrm{HH}$ pathway dependence of lung cancer cells (37). It was therefore not unexpected to detect prominent Gli1 immunostaining of lung tumors arising from ED-1 cells after their tail-vein injections into syngeneic FVB mice (data not shown).

To explore independently Smo inhibitor effects on lung cancer cells, ED-1 cells were engineered to have Smo knockdown. This was done using two independent shRNAs versus an inactive control shRNA (Fig. 4E). This conferred significant repression (Smo1 shRNA $\mathrm{P}=0.002$ and $\mathrm{Smo} 2$ shRNA $\mathrm{P}=0.001$ ) of transplanted lung cancers (Fig. 4F). Ex vivo treatment of ED-1 cells with another Smo inhibitor MK-4101 significantly reduced ED-1 colony formation ( $\mathrm{P}<0.01$, data not shown) and tumorigenicity $(\mathrm{P}=0.009)$ after tail-vein injections into syngeneic mice of these versus control cells (Fig. 4G).

Cyclopamine responses were examined in cyclin E transgenic mice because their lung cancers expressed a gene profile that was indicative of dependence on $\mathrm{HH}$ signaling. Cyclopaminetreatment decreased immunohistochemical expression of the proliferation markers Ki-67, cyclin D1 and cyclin E in the neoplastic versus normal lungs of treated versus age and sexmatched control mice (Fig. 5A, left panel). This result suggested that Smo inhibition would also reduce lung tumor formation in 

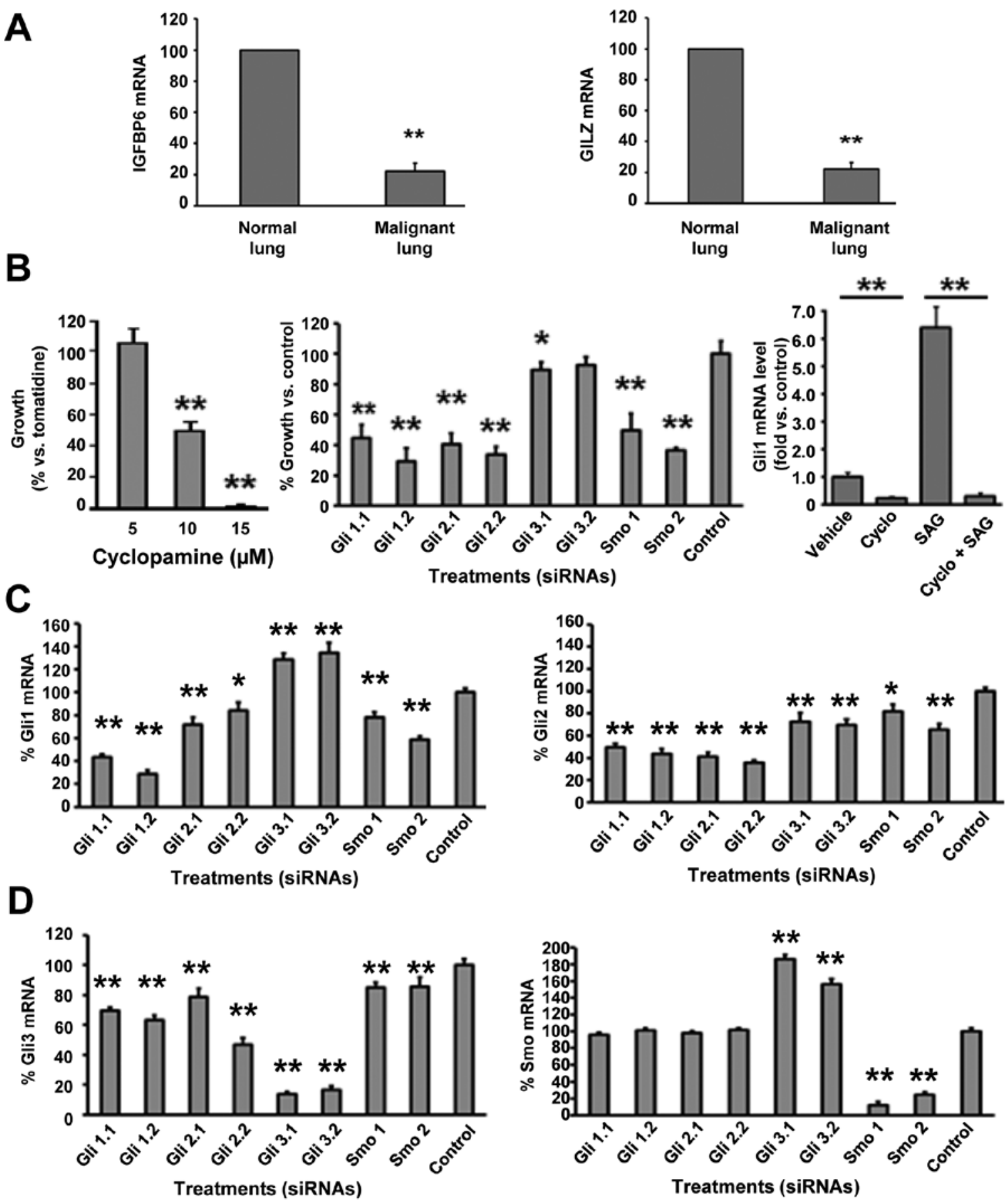

Figure 3. Hedgehog $(\mathrm{HH})$ pathway member expression and regulation in lung cancers and lung cancer cell lines. (A) Cyclin E transgenic lung cancers expressed lower levels of IGFBP6 versus the adjacent normal lung tissue (left panel). Cyclin E transgenic lung cancers expressed lower mRNA levels of GILZ as compared to the adjacent normal lung tissue (right panel). These data are representative results of three triplicate replicate experiments. (B) ED-1 cells responded to pharmacologic (left panel) or siRNA-mediated (middle panel) repression of HH pathway members. Gli1 mRNA levels are shown 3 days after cyclopamine (cyclo, $10 \mu \mathrm{M}$ ), SAG (50 nM), or combined treatments (right panel). (C and D) Validation of individual Gli1, Gli2, Gli3, or Smo knock-downs in ED-1 cells. Compensatory effects on $\mathrm{HH}$ pathway members are shown. ${ }^{*} \mathrm{P}<0.05 ;{ }^{* *} \mathrm{P}<0.01$. Standard deviation bars are displayed.

the transplantation model. ED-1 cells were injected into the tail veins of syngeneic mice. Cyclopamine treatment of mice began after lung tumors were histologically present (data not shown). This significantly $(\mathrm{P}=0.00002)$ reduced lung cancers in cyclopamine versus vehicle-treated mice (Fig. 5A, right panel).

To learn whether a profile indicative of $\mathrm{HH}$ pathway dependence was expressed in human lung cancers, a normal-malignant lung tissue array (20) was studied. Immunohistochemical expression profiles revealed significant differences in the malignant versus normal lung for cyclin $\mathrm{E}(\mathrm{P}<0.01)$, IGFBP6 $(\mathrm{P}<0.01)$, and GILZ $(\mathrm{P}<0.01)$ in Fig. 5B. Lung cancers with high cyclin $\mathrm{E}$ levels expressed significantly $(\mathrm{P}<0.01)$ higher levels of Gli1 than did cases with reduced cyclin E levels (Fig. 5B). Cases with high cyclin $\mathrm{E}$ and low IGFBP6 levels had increased $(\mathrm{P}<0.0001)$ Gli1 immunohistochemical expression. Representative results are presented (Fig. 5C). 

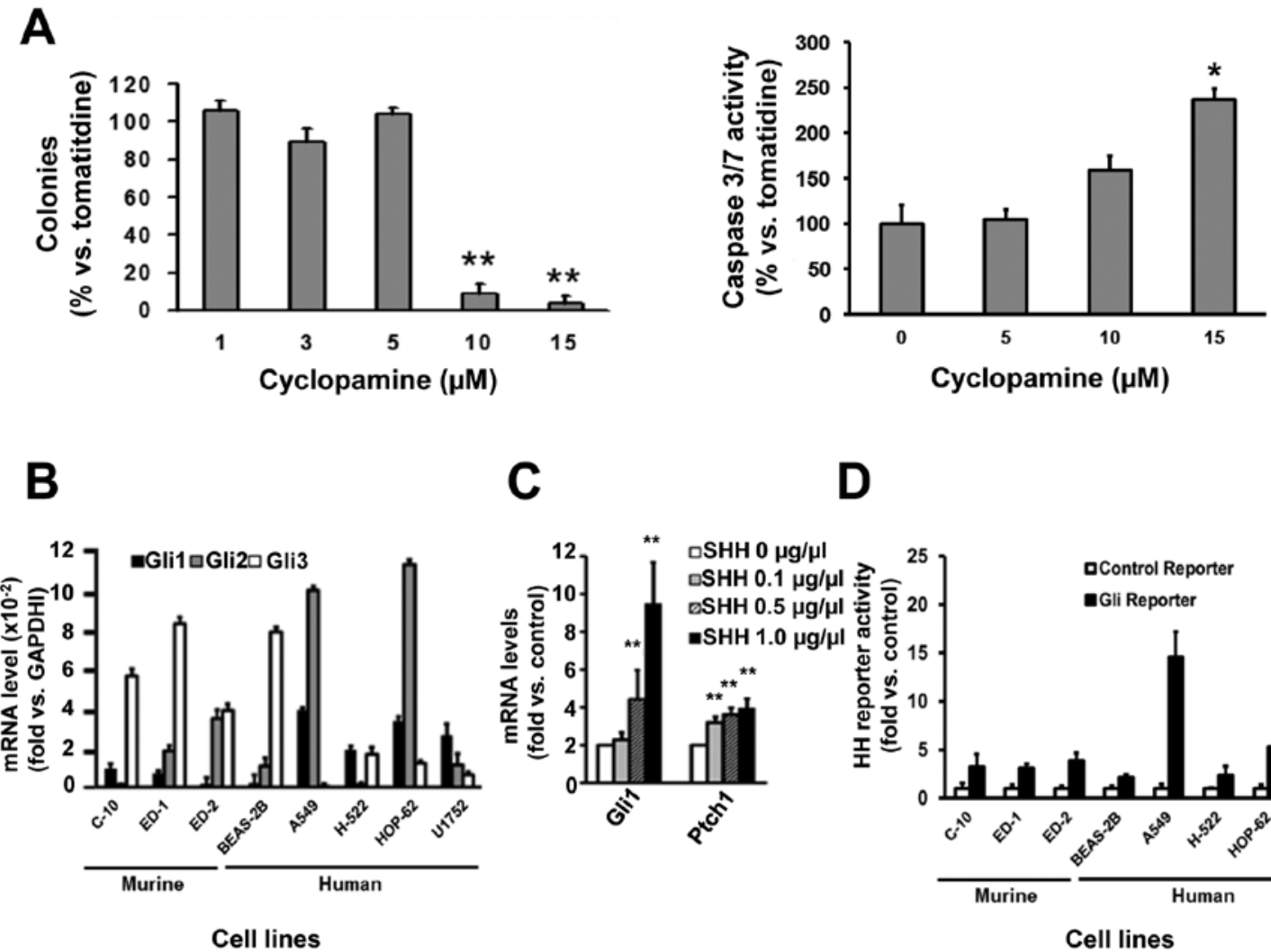

D

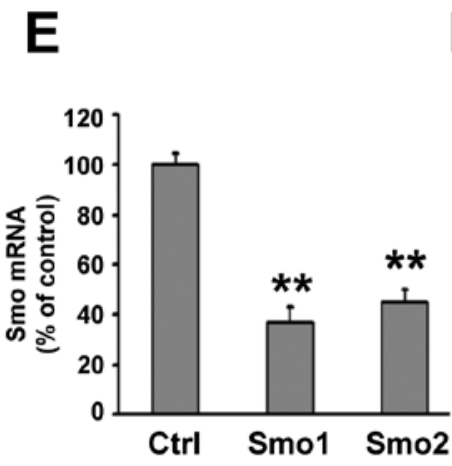

$\mathbf{F}$

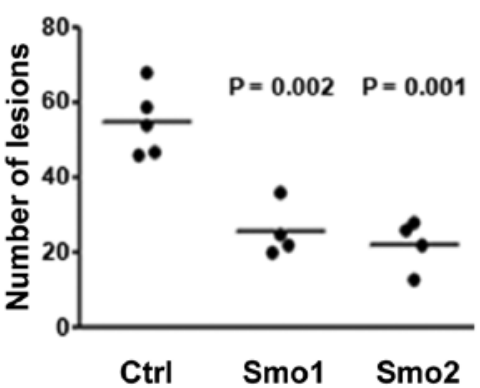

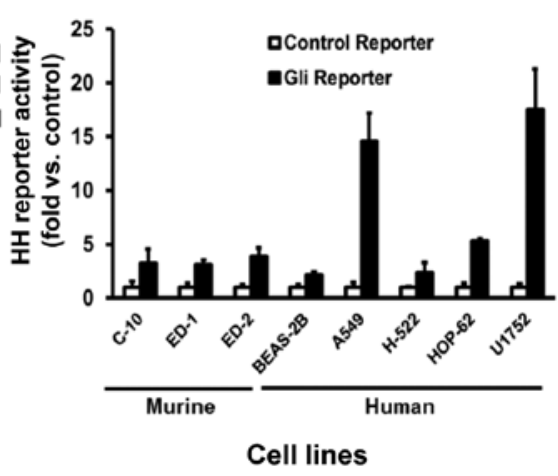

$\mathbf{G}$

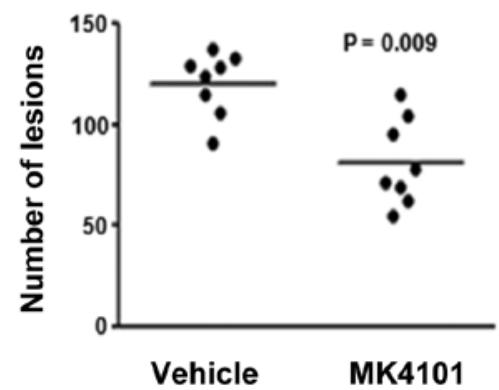

Figure 4. Expression of $\mathrm{HH}$ pathway members in immortalized pulmonary epithelial and lung cancer cell lines. Effects of Smo inhibition on lung cancer cell clonal growth, apoptosis and lung tumor formation were also examined. (A) Cyclopamine treatment of ED-1 cells significantly inhibited clonal growth of this lung cancer cell line (left panel). This treatment also increased apoptosis as scored by caspase 3/7 activity (right panel). In these panels, dose-responsive effects are displayed with comparisons made to tomatidine treatments. (B) ED-1 and ED-2 lung cancer cells expressed multiple HH pathway members. Findings were compared to C-10 immortalized murine lung epithelial cells. Analogous experiments were performed using BEAS-2B immortalized human bronchial epithelial cells and human lung cancer cell lines. The mRNA levels are shown. (C) Recombinant sonic hedgehog (sHH) significantly induced mRNA levels of the indicated HH regulated species in ED-1 cells. (D) Gli-BS-luciferase reporter activity in murine and human immortalized lung epithelial and lung cancer lines. (E) Repression of Smo by independent transfection of shRNAs (Smo1 and Smo2) versus controls. (F) Significant reduction of lung cancer formation in vivo after transplantation of ED-1 cells that express Smo1 or Smo2 shRNAs versus control shRNA. Each circle represents an individual mouse; horizontal lines indicate mean tumor numbers. (G) Ex vivo treatment of ED-1 cells with the Smo inhibitor MK-4101 before tail vein injection into syngeneic mice also reduced lung tumor formation. Each circle represents an individual mouse; horizontal lines indicate mean tumor numbers. Standard deviation bars are shown. ${ }^{*} \mathrm{P}<0.05 ;{ }^{* *} \mathrm{P}<0.01$.

\section{Discussion}

Smo inhibitors are active against Gorlin syndrome-associated BCC or medulloblastoma where Ptch mutations occur (9-13). Smo mutations confer resistance to Smo inhibitors $(17,18)$. This study comprehensively interrogated 705 epithelial cancer cell lines for growth response to the Smo inhibitor cyclopamine. Findings were compared with expressed HH pathway-regulated species using a linked genetic database. Ptch and Smo mutations that respectively conferred Smo inhibitor response or resistance were undetected. Rare variant sequences were found, but their functional impact was not established. Because $\mathrm{HH}$ pathway activation occurs in lung cancers, findings were validated using different Smo inhibitors in human lung cancer cell lines, transgenic and transplantable murine lung cancer models, and paired normal-malignant lung tissue arrays. Ptch1 or Smo mutations were undetected in examined murine and human immortalized lung epithelial and cancer cell lines. 

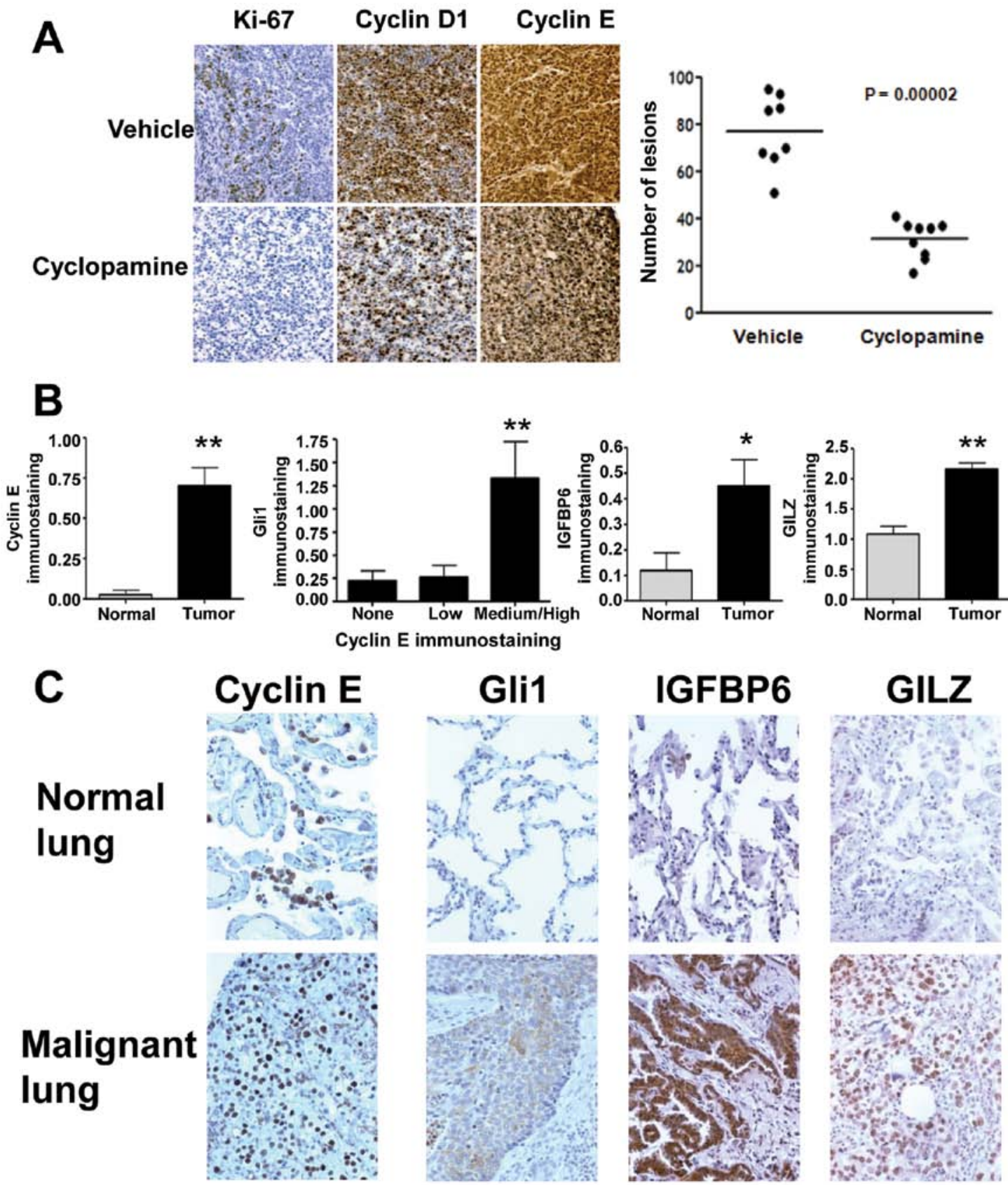
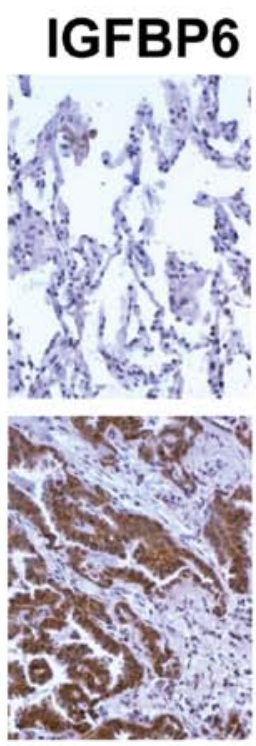
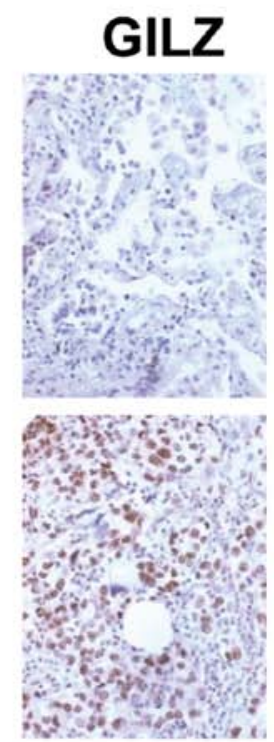

Figure 5. Cyclopamine treatment reduced proliferation and lung tumor formation in transgenic cyclin E mouse models. The expression profiles of HH pathway members in paired human malignant-normal lung tissues are displayed. (A) Decreased immunohistochemical detection of Ki-67, cyclin D1 and cyclin E in representative lung adenocarcinomas of transgenic cyclin E mice treated with cyclopamine versus vehicle controls (left panel). Cyclopamine significantly $(\mathrm{P}=0.00002)$ decreased lung cancers in the described murine syngeneic transplantable lung cancer model (right panel). Each circle represents an individual mouse; horizontal lines display mean tumor numbers. (B) Cyclin E, IGFBP6, and GILZ were each significantly (P<0.01) differentially expressed in human lung cancer versus normal lung. Cancers with high cyclin E immunostaining expressed high Glil levels (P<0.01). (C) Individual immunohistochemical expression for cyclin E, Gli1, IGFBP6, and GILZ in representative human normal versus malignant lung tissues. ${ }^{*} \mathrm{P}<0.05 ;{ }^{* *} \mathrm{P}<0.01$.

Smo inhibitor growth response was most significantly associated with high cyclin $\mathrm{E}(\mathrm{P}=0.000009)$, low IGFBP6 $(\mathrm{P}=0.000004)$, and high Gli1 ( $\mathrm{P}=0.04)$ levels; high GILZ levels were associated with reduced response $(\mathrm{P}=0.002)$ in Fig. 1. This profile implicated a basal dependence on the HH pathway for the growth of these cancer cells. This possibility was validated in murine and human lung cancer cell lines and transgenic as well as transplantable murine lung cancer models in Figs. 1-5. Functional consequences were shown using several pharmacological and genetic Smo inhibitors.
Differential expression of the same species was observed in a malignant versus normal human lung tissue array (Fig. 5). A profile indicative of Smo inhibitor response was also observed in murine lung cancers (Fig. 3). While high IGFBP6 levels were present in some lung tumors, it is notable that lung cancers with both high cyclin E and reduced IGFBP6 expression significantly $(\mathrm{P}<0.0001)$ increased Gli1 expression. This pattern was one indicating response to a Smo inhibitor. This points out the potential clinical need to discern profiles of $\mathrm{HH}$ pathway-regulated species in human tumors to learn which are likely to be responsive to 
a Smo inhibitor. Future clinical trials that explore activity of Smo inhibitors should also determine these profiles to uncover possible $\mathrm{HH}$ pathway dependence.

Several Smo inhibitors conferred similar effects. Stromal effects are engaged to confer some of these anti-neoplastic effects. This could account for differences between in vitro and in vivo effects of Smo inhibition $(37,38)$. The findings presented in Fig. 1 could explain why trials with Smo inhibitors might underestimate clinical anti-tumor effects of Smo inhibition. Only some cancers express a gene profile indicating possible $\mathrm{HH}$ pathway dependence. Notably, autocrine $\mathrm{HH}$ pathway signaling occurs in lung cancers (36). The findings reported here are consistent with this prior study.

These findings have implications for combination cancer therapy. Responses to Smo inhibition occurred whether or not $R A S$ or $p 53$ (or Ptchl or Smo) mutations were present in cancer cells (Table I). In clinical lung cancers, $K-R A S$ mutations confer resistance to EGFR-TKIs $(32,34,35)$. Yet, clinical trials revealed activity against lung cancers having $K-R A S$ mutations when an EGFR-TKI was combined with a rexinoid (33). This clinical activity was associated with reduced cyclin D1 expression in posttreatment lung cancer biopsies (33). In the present study, in vivo responses to Smo inhibition were linked to cyclin D1 repression (Fig. 5). Adding a Smo inhibitor to a regimen that targets cyclin D1 for repression might enhance clinical anti-tumor activity. Smo responses were also associated with cyclin E expression (Figs. 1 and 5). Targeting the cyclin E-cdk2 complex exerted anti-tumor responses despite presence of $K-R A S$ mutations by inducing anaphase catastrophe $(19,39)$. Combining a cdk2 antagonist with a Smo inhibitor might augment anti-neoplastic activity.

High Smo inhibitor dosages were associated with these anti-neoplastic effects. A similar dose-response relationship for $\mathrm{HH}$ inhibition was observed in different tumor contexts and this might depend on expressed drug transporters (40,41). It is notable that the findings displayed in Fig. 3B (right panel) using a Smo agonist argue against off-target effects of cyclopamine. The reduction of lung tumors after Smo inhibition in the transplantation model reported here is notable since these lung cancers did not have Ptch1 or Smo mutations. Smo inhibitors might treat or prevent other cancers that lack these mutations.

Taken together, findings presented here indicate a Smo inhibitor should be considered in cancers that lack Smo or Ptch1 mutations. This is especially the case when the tumors express a gene profile indicating basal activation of the $\mathrm{HH}$ pathway. This could implicate a dependence on the $\mathrm{HH}$ pathway for growth or survival of the same tumors. Future clinical research should explore the translational consequences of these findings for cancer therapy and prevention.

\section{Acknowledgements}

This study was supported by National Institutes of Health (NIH) and National Cancer Institute (NCI) grants R01-CA087546 (E.D.), R01-CA111422 (E.D.), R03-CA132166 (E.D.), a Samuel Waxman Cancer Research Foundation award (E.D.), a research grant from Merck (E.D.) and an American Cancer Society Clinical Research Professorship (E.D.) supported by a gift from the FM Kirby Foundation. A.M.B. was supported by an NIH National Research Service Award (T32-CA009658). We thank Dr Jason Sparkowski (Merck) for providing MK-4101.

\section{References}

1. Ingham PW and McMahon AP: Hedgehog signaling in animal development: paradigms and principles. Genes Dev 15: 3059-3087, 2001.

2. Pasca di Magliano M and Hebrok M: Hedgehog signaling in cancer formation and maintenance. Nat Rev Cancer 3: 903-911, 2003.

3. Zhu H and Lo H-W: The human glioma-associated oncogene homolog 1 (GLI1) family of transcription factors in gene regulation and diseases. Curr Genomics 11: 238-245, 2010.

4. Ingram WJ, Wicking CA, Grimmond SM, Forrest AR and Wainwright BJ: Novel genes regulated by sonic hedgehog in pluripotent mesenchymal cells. Oncogene 21: 8196-8205, 2002.

5. Duman-Scheel M, Weng L, Xin S and Du W: Hedgehog regulates cell growth and proliferation by inducing cyclin $\mathrm{D}$ and cyclin $\mathrm{E}$. Nature 417: 299-304, 2002.

6. Watkins DN, Berman DM, Burkholder SG, Wang B, Beachy PA and Baylin SB: Hedgehog signaling within airway epithelial progenitors and in small-cell lung cancer. Nature 422: 313-317, 2003.

7. Kubo M, Nakamura M, Tasaki A, et al: Hedgehog signaling pathway is a new therapeutic target for patients with breast cancer. Cancer Res 64: 6071-6074, 2004.

8. Thayer SP, di Magliano MP, Heiser PW, et al: Hedgehog is an early and late mediator of pancreatic cancer tumorigenesis. Nature 425 : 851-856, 2003.

9. Rudin CM, Hann CL, Laterra J, et al: Treatment of medulloblastoma with hedgehog pathway inhibitor GDC-0449. N Engl J Med 361: 1173-1178, 2009.

10. Caro I and Low JA: The role of the hedgehog signaling pathway in the development of basal cell carcinoma and opportunities for treatment. Clin Cancer Res 16: 3335-3339, 2010.

11. Von Hoff DD, LoRusso PM, Rudin CM, et al: Inhibition of the hedgehog pathway in advanced basal-cell carcinoma. N Engl J Med 361: 1164-1172, 2009.

12. Low JA and de Sauvage FJ: Clinical experience with hedgehog pathway inhibitors. J Clin Oncol 28: 5321-5326, 2010.

13. Yang JY and Marghoob AA: Emerging treatments and signaling pathway inhibitors. Semin Cutan Med Surg 31: S14-S18, 2011.

14. Chen JK, Taipale J, Cooper MK and Beachy PA: Inhibition of hedgehog signaling by direct binding of cyclopamine to smoothened. Genes Dev 16: 2743-2748, 2002.

15. Yuan Z, Goetz JA, Singh S, et al: Frequent requirement of hedgehog signaling in non-small cell lung carcinoma. Oncogene 26: 1046-1055, 2007.

16. Ma Y, Fiering S, Black C, et al: Transgenic cyclin E triggers dysplasia and multiple pulmonary adenocarcinomas. Proc Natl Acad Sci USA 104: 4089-4094, 2007.

17. Dijkgraaf GJP, Alicke B, Weinmann L, et al: Small molecule inhibition of GDC-0449 refractory smoothened mutants and downstream mechanisms of drug resistance. Cancer Res 71: 435-444, 2011.

18. Yauch RL, Dijkgraaf GJP, Alicke B, et al: Smoothened mutation confers resistance to a hedgehog pathway inhibitor in medulloblastoma. Science 326: 572-574, 2009.

19. Galimberti F, Thompson SL, Liu X, et al: Targeting the cyclin E-cdk-2 complex represses lung cancer growth by triggering anaphase catastrophe. Clin Cancer Res 16: 109-120, 2010.

20. Liu X, Sempere LF, Ouyang H, et al: MicroRNA-31 functions as an oncogenic microRNA in mouse and human lung cancer cells by repressing specific tumor suppressors. J Clin Invest 120: 1298-1309, 2010

21. Liu X, Sempere LF, Galimberti F, et al: Uncovering growthsuppressive microRNAs in lung cancer. Clin Cancer Res 15: 1177-1183, 2009.

22. Gupta S, Takebe $N$ and Lorusso P: Targeting the hedgehog pathway in cancer. Ther Adv Med Oncol 2: 237-250, 2010.

23. McDermott U, Sharma SV, Dowell L, et al: Identification of genotype-correlated sensitivity to selective kinase inhibitors by using high-throughput tumor cell line profiling. Proc Natl Acad Sci USA 104: 19936-19941, 2007.

24. McDermott U, Sharma SV and Settleman J: High-throughput lung cancer cell line screening for genotype-correlated sensitivity to an EGFR kinase inhibitor. Methods Enzymol 438: 331-341, 2008.

25. R: Test for Association/Correlation Between Paired Samples. http:// stat.ethz.ch/R-manual/R-patched/library/stats/html/cor.test.html

26. rproject.org. http://www.rproject.org/

27. GSK Cancer Cell Line Genomic Profiling Data. https://cabig.nci. nih.gov/caArray_GSKdata/ 
28. Bamford S, Dawson E, Forbes S, et al: The COSMIC (catalogue of somatic mutations in cancer) database and website. Br J Cancer 91: 355-358, 2004

29. Lindström E, Shimokawa T, Toftgård R and Zaphiropoulos PG: PTCH mutations: distribution and analyses. Hum Mutat 27 215-219, 2006.

30. Freemantle SJ and Dmitrovsky E: Cyclin E transgenic mice: discovery tools for lung cancer biology, therapy, and prevention. Cancer Prev Res 3: 1513-1518, 2010.

31. Feng Q, Sekula D, Guo Y, et al: UBE1L causes lung cancer growth suppression by targeting cyclin D1. Mol Cancer Ther 7: 3780-3788, 2008.

32. Massarelli E, Varella-Garcia M, Tang X, et al: KRAS mutation is an important predictor of resistance to therapy with epidermal growth factor receptor tyrosine kinase inhibitors in non-smallcell lung cancer. Clin Cancer Res 13: 2890-2896, 2007.

33. Dragnev KH, Ma T, Cyrus J, et al: Bexarotene plus erlotinib suppress lung carcinogenesis independent of KRAS mutations in two clinical trials and transgenic models. Cancer Prev Res 4 818-828, 2011.

34. Linardou H, Dahabreh IJ, Kanaloupiti D, et al: Assessment of somatic k-RAS mutations as a mechanism associated with resistance to EGFR-targeted agents: a systematic review and metaanalysis of studies in advanced non-small-cell lung cancer and metastatic colorectal cancer. Lancet Oncol 9: 962-972, 2008.
35. Pao W, Wang TY, Riely GJ, et al: KRAS mutations and primary resistance of lung adenocarcinomas to gefitinib or erlotinib. PLoS Med 2: e17-e17, 2005.

36. Singh S, Wang Z, Liang Fei D, et al: Hedgehog-producing cancer cells respond to and require autocrine hedgehog activity. Cancer Res 71: 4454-4463, 2011.

37. Vestergaard J, Pedersen MW, Pedersen N, et al: Hedgehog signaling in small-cell lung cancer: frequent in vivo but a rare event in vitro. Lung Cancer 52: 281-290, 2006.

38. Chen JK, Taipale J, Young KE, Maiti T and Beachy PA: Smal molecule modulation of smoothened activity. Proc Natl Acad Sci USA 99: 14071-14076, 2002.

39. Galimberti F, Thompson SL, Ravi S, Compton DA and Dmitrovsky E: Anaphase catastrophe is a target for cancer therapy. Clin Cancer Res 17: 1218-1222, 2011.

40. Sims-Mourtada J, Izzo JG, Ajani J and Chao KSC: Sonic hedgehog promotes multiple drug resistance by regulation of drug transport. Oncogene 26: 5674-5679, 2007.

41. Tang T, Tang JY, Li D, et al: Targeting superficial or nodular basal cell carcinoma with topically formulated small molecule inhibitor of smoothened. Clin Cancer Res 17: 3378-3387, 2011. 